\title{
The role of CAMP signaling in the regulation of interleukin-33 production by RAW264.7 macrophages in response to various doses of lipopolysaccharide
}

\author{
Yoshiki Yanagawa, Shizuka Sato, Sachiko Hiraide, Kenji lizuka \\ Department of Pharmacology, School of Pharmaceutical Sciences, Health Sciences University of Hokkaido, Japan
}

Background: Recent reports suggest that interleukin (IL)-33 plays pathogenic roles in various inflammatory disorders including allergic diseases. However the mechanism underlying regulation of IL-33 production remains poorly understood. At present, we examined the role of cAMP signaling in the regulation of IL-33 production by RAW264.7 macrophages in response to various doses of lipopolysaccharide (LPS).

Methods: RAW264.7 cells were obtained from ATCC and cultured in 5\% FBS RPMI-1640 medium. 8-Br-cAMP or adrenaline was used at $100 \mu \mathrm{M}$ or $1 \mu \mathrm{M}$. Amount of IL-33 and tumor necrosis factor (TNF) protein was quantitated by ELISA. Level of IL-33 and TNF mRNA was determined by quantitative RT-PCR.

Results: High-dose LPS significantly induced IL-33 and TNF protein production in RAW264.7 macrophages. In contrast, low-dose LPS showed little effect on IL-33 production, whereas significantly inducing TNF production. In the presence of the membrane-permeable cAMP analog 8-Br-cAMP, low-dose LPS was able to induce vigorous IL-33 production. This phenomenon was consistent with mRNA levels. Similarly, the cAMP-elevating agent adrenaline also enhanced the sensitivity of RAW264.7 macrophages to LPS with respect to IL-33 production but not TNF production. Agonistantagonist studies have verified that adrenaline-induced IL-33 production was mainly mediated via $\beta_{2}$-adrenoceptor. The protein kinase A (PKA) inhibitor H89 blocked the effects of 8-Br-cAMP and adrenaline on IL-33 production, suggesting an involvement of PKA in IL-33 induction.

Conclusion: Taken together, cAMP signaling induced by cAMP-elevating agents, such as adrenaline, appears to enhance the sensitivity of RAW264.7 macrophages to LPS with respect to IL-33 production. 